\title{
Schlechtere Kontrolle bei Sinusitis?
}

Matsuno $\mathrm{O}$ et al. Asthma and sinusitis: association and implication.

Int Arch Allergy Immunol. 2008;147:52-58

\section{Hintergrund und Fragestellung}

In einer großen deutschen prospektiven Querschnittsuntersuchung lagen bei 56\% der Patienten mit chronischer Sinusitis weitere Atemwegserkrankungen vor [1]:

-Allergische Rhinitis: 17,3\%

_Asthma bronchiale: 12,0\%

— Bronchitis: 16,8\%

- Septumdeviation: $17,3 \%$

-Allergien/Noxen: 21,2\%.

Somit treten Sinusitiden auch bei Asthmatikern gehäuft auf. Sinusitiden sind bei Asthmatikern häufiger chronisch entzündliche Veränderungen der paranasalen Mukosa als akute bakterielle Sinusitiden.

Die bei Asthma bronchiale häufig vorliegende allergische Rhinitis stellt einen Risikofaktor für eine Sinusitis dar. Inwieweit umgekehrt eine Sinusitis einen Risikofaktor für ein schwergradiges Asthma bronchiale darstellt, ist umstritten.

\section{Methodik}

Eine japanische Studie untersuchte die Prävalenz von Symptomen einer Rhinitis oder Sinusitis bei 188 ambulant behandelten Patienten mit mäßig- bis schwergradigem Asthma bronchiale.
Über drei Monate hinweg wurden die Asthmapatienten von Pneumologen und HNO-Ärzten untersucht und der Krankheitsverlauf beobachtet. Patientenfragebögen, die jeweils die zurückliegende Woche erfassten, ergänzten die Informationen über das zeitliche Auftreten und den Schweregrad des Asthma und der Sinusitis.

\section{Ergebnisse}

Von den untersuchten Asthmapatienten litten

-37\% gleichzeitig an einer Sinusitis,

$-56 \%$ gleichzeitig an einer Rhinitis,

$-19 \%$ an einer Otitis media,

$-13 \%$ an Nasenpolypen.

Bei 104 Patienten wurde eine Computertomografie (CT) der Nasennebenhöhlen (NNH) durchgeführt. 66\% von ihnen wiesen einen pathologischen NNHBefund auf. Auch 18\% der Asthmapatienten ohne anamnestischen Hinweis auf eine Sinusitis zeigten im NNH-CT einen pathologischen Befund.

Eine signifikante Korrelation zwischen dem Schweregrad des Asthma bronchiale und dem der Sinusitis (NNH-CT) konnte überraschenderweise nicht nachgewiesen werden. Asthmapatienten ohne begleitende Sinusitis hatten jedoch signifi-

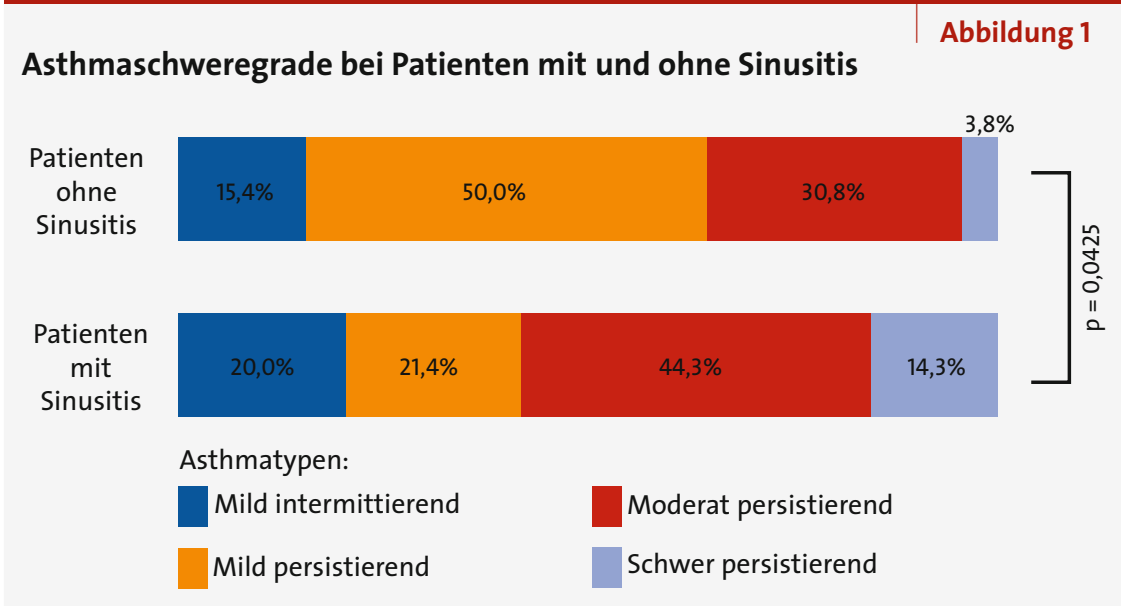

kant niedrigere Asthmaschweregrade ( $\mathrm{p}$ = 0,0425). Bei Asthmapatienten mit stark ausgeprägter Sinusitis traten signifikant häufiger Asthmaanfälle auf. Bei Erwachsenen ging der Asthmadiagnose in 69\% der Fälle eine Sinusitis voraus, während dies bei Kindern nicht der Fall war.

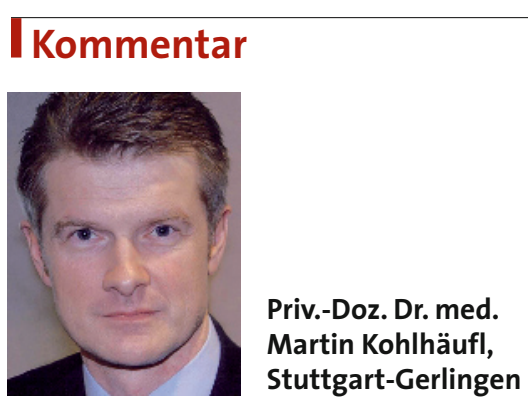

Sinunasale Symptome sind bei Asthmapatienten häufig, jedoch nicht spezifisch für eine chronische sinunasale Erkrankung. Die Koinzidenz von Asthma bronchiale und Sinusitis wurde in dieser japanischen Studie bei $37 \%$ der Patienten nachgewiesen, was die Ergebnisse der o.g. prospektiven deutschen Untersuchung bestätigt [1]. Die Assoziation zwischen Asthma bronchiale und Sinusitis ist somit eng und in den meisten Fällen klinisch relevant. Die hohe Koprävalenz von Asthma und Sinusitis legt nahe, dass der von der allergischen Rhinitis bekannte „Etagenwechsel“ bei der Sinusitis ebenfalls vorkommen kann.

Zur adäquaten Asthmakontrolle ist die Mitbehandlung von Rhinitis und Sinusitis zwingend erforderlich. Sie kann den Verbrauch an Asthmamedikation signifikant vermindern.

Als praxisnaher Surrogatmarker für eine bakterielle Genese wird eine Beschwerdedauer von mindestens zehn Tagen oder eine Verschlimmerung nach fünf Tagen betrachtet. Die Deutsche Gesellschaft für Hals-Nasen-Ohren-Heilkunde (DGHNO) empfiehlt zur Behandlung der Sinusitis eine Tripletherapie. Diese besteht aus der Kombination von Antibiotikum (ohne konkrete Empfehlungen von Substanzklassen), Analgetikum und Vasokonstriktivum. 